\title{
Estrategias para reducir la ansiedad durante las actividades orales en estudiantes de inglés
}

\author{
Damaris Cordero Badilla \\ Escuela de Literatura y Ciencias del Lenguaje \\ Universidad Nacional, Costa Rica \\ Yuri Morales Rojas \\ Escuela de Literatura y Ciencias del Lenguaje \\ Universidad Nacional, Costa Rica
}

\begin{abstract}
Resumen
Uno de los principales problemas que un profesor de lengua puede enfrentar en relación con el aprendizaje de idiomas es la ansiedad, factor afectivo que tiene un gran impacto en la comunicación oral de los estudiantes durante las lecciones. Debido a eso, una investigación sobre el nivel de ansiedad que los estudiantes pudieran tener mientras llevan a cabo las actividades orales en inglés se considera importante, con el fin de encontrar posibles técnicas o estrategias que ayuden a que los estudiantes se sientan más seguros al utilizar esta segunda lengua para comunicarse con sus compañeros de clase o con sus profesores. Con este objetivo en mente y para recolectar la información que serviría de apoyo a este trabajo, se realizó una investigación de tipo cuantitativo y un diseño de tipo cuasiexperimental. Una vez analizada la información, se concluyó que el uso de las estrategias para reducir la ansiedad con estos estudiantes en un periodo de cuatro meses fue altamente efectivo, según la opinión expresada por los mismos estudiantes y las observaciones implementadas por las profesoras a cargo de estos grupos de alumnos.
\end{abstract}

Palabras claves: ansiedad, comunicación oral, estrategias para la reducción de la ansiedad 


\begin{abstract}
One of the main problems that a language teacher may face in relation to language learning is anxiety, which is one of the emotional factors that have a great impact in the oral communication of the students during the lessons. Because of that, an investigation about the level of anxiety that students may have while performing oral English activities is deemed important in order to find possible techniques or strategies to help them feel more secure when using this second language to communicate with their classmates or their teachers. With this objective in mind and to gather the information that would support this work it was thought to make a Quantitative research and a Quasiexperimental design. Having analyzed the information it was concluded that the use of strategies to reduce the anxiety with these students and in a period of 4 months were highly effective, according to the opinion expressed by the students and the observations implemented by the teachers in charge of these groups.
\end{abstract}

Keywords: anxiety, oral communication, strategies for reducing anxiety

\section{Introducción}

I nglés I para otras carreras en la Universidad Nacional es uno de los cursos que se financia con un presupuesto proporcionado por el Consejo Nacional de Rectores de Costa Rica (Conare) a cada institución de educación superior pública, como una manera de mejorar el nivel de inglés de los estudiantes de las diferentes carreras que ofrecen estas instituciones. Debido a esto, se ha establecido que, al final del semestre y de acuerdo con el Marco Común Europeo de Referencia (MCER), los estudiantes lleguen a alcanzar un nivel A1 en las cuatro destrezas lingüísticas en inglés (lectura, escritura, comprensión auditiva y expresión oral).

No obstante, los estudiantes de Inglés I para otras carreras de la UNA llegan a esta institución de educación superior provenientes de escuelas secundarias costarricenses públicas y privadas, y en muchos casos con un nivel de inglés que no es el requerido en la Universidad Nacional para este tipo de cursos. En consecuencia, los docentes planean las actividades para las clases de inglés con un propósito intrínseco de hacer que los estudiantes hablen; no obstante, perciben problemas afectivos que influyen en la disposición a participar en las actividades orales. En este momento, se buscan las posibles causas e información sobre técnicas que ayuden a que se sientan seguros al hablar con sus compañeros y con las profesoras durante las lecciones. Esto con el objetivo de cumplir con lo que estipula el MCER en uno de sus apartados en cuanto a que al final del semestre el estudiante será capaz de:

Comunicarse en tareas sencillas y habituales, con el simple intercambio de información y comprender lo suficiente como para mantener la conversación, utilice una serie de expresiones y frases para describir su familia, condiciones, antecedentes educativos y trabajo actual o reciente viviente. (Pearson, 2013, p.8) 
Por lo tanto, y con este fin, los educadores preparan actividades orales como dramatizaciones, interacciones grupales, discusiones y otras en las que los aprendices se involucran conversando y participan de forma individual y en grupos con el resto de los compañeros de clase. Pero, esta es la parte más difícil en muchos casos porque hay cierta resistencia a participar en estas actividades y cierto nivel de ansiedad se percibe cuando se les pide que lo hagan. Por esta razón, se consideró importante hacer un análisis del nivel de ansiedad que los estudiantes pudieran tener durante las actividades orales y las posibles causas, así como las estrategias de enseñanza que ayuden a reducir este sentimiento en ellos.

\section{Revisión de literatura}

Las emociones, los sentimientos y la ansiedad son algunos de los dominios afectivos que, según Oxford (1990) y White (2003), influyen en la disposición y actitud de un estudiante en el aprendizaje de una lengua extranjera. Debido a eso, cada maestro debe tener en cuenta estas causas si quiere mantener a los alumnos motivados y en la tarea durante las lecciones.

Los estudiantes en un aula de lenguas extranjeras están expuestos a diferentes experiencias de enseñanza y aprendizaje, y se pretende que las lleven a cabo con éxito. No obstante, algunos de ellos podrían tener un mal desempeño debido a que no se sienten seguros de sí mismos cuando utilizan esta segunda lengua. He aquí la importancia de averiguar sobre las causas que puedan intervenir en la disposición que tengan de interactuar en forma oral con sus compañeros de clase o con sus profesoras.

La ansiedad ha sido una de las emociones afectivas que ha llamado la atención a investigadores en el campo de la psicología; por tratarse de un factor afectivo, interfiere en el proceso de aprendizaje en el contexto de aulas de clases donde se adquiere un segundo idioma. Seguidamente se presentan los aspectos más relevantes relacionados con la ansiedad, como su definición y factores que la originan, así como estrategias para facilitar a los y las docentes el proceso de enseñanza-aprendizaje de una segunda lengua.

\section{La ansiedad}

La salud mental interviene de modo representativo en la calidad de vida y significativamente favorece una plena participación social de los individuos.

La ansiedad es una emoción que aparece ante situaciones que representan peligro; prepara al sujeto para proceder ante ellas mediante una serie de reacciones cognitivas, fisiológicas y conductuales. Las personas que sufren altos niveles de ansiedad no suelen adaptarse adecuadamente, lo que da como resultado alarmas ante supuestas amenazas factibles; en otras ocasiones, tienden a percibir con frecuencia una serie de amenazas que incitan niveles excesivamente altos de ansiedad.

La ansiedad involucra tres métodos de respuesta la subjetiva-cognitiva, que es la percepción y la evaluación subjetiva de los estímulos externos experimentados de forma desagradable por el individuo, como la preocupación, la inquietud, el miedo, el pánico. El modo fisiológico-somático implica la activación 
del sistema nervioso autónomo asociado con la experiencia de ansiedad como la sudoración, el temblor, el incremento de la tensión muscular, el aumento de la frecuencia cardiaca y de la tensión muscular, la aceleración respiratoria, y las náuseas, entre otros. El componente motor-conductual es el escape o la evitación ante situaciones amenazantes y posee componentes observables de la conducta del individuo como movimientos, posturas corporales y expresiones faciales.

De acuerdo con la Revista Médica de Costa Rica y Centroamérica (LXIX, 604, 2012, 497-507), los trastornos de ansiedad son, como grupo, las enfermedades mentales más comunes, con una alta proporción de aproximadamente $2-8 \%$ en la población general y $10-12 \%$ en las consultas de atención primaria. En los últimos años, se ha observado un incremento, así como de las enfermedades y efectos que a menudo los siguen. Los trastornos de ansiedad son dos veces más prevalentes en mujeres. Se calcula que uno de diez pacientes que se atienden diariamente presenta un trastorno de ansiedad, siendo incluso más común que la diabetes mellitus.

El origen de los trastornos de ansiedad constituye un patrón donde interactúan múltiples factores: biológicos, psicológicos por motivaciones o impulsos, sociales, traumáticos y de aprendizaje. Junto a esta etiología, se menciona la presencia de factores de riesgo y protectores que intervienen en la manifestación de este trastorno. Según Bustamante (2013), neurológicamente, la ansiedad tiene su origen en ciertas zonas del tallo cerebral, que están implicadas en el desarrollo y producción de la angustia, y el hipocampo y la amígdala pertenecientes al sistema límbico. El sistema Gaba es el neurotransmisor de acción inhibitoria más significativo, pues funciona como ansiolítico o como generador de angustia. El sistema serotoninérgico es igualmente importante, pues está en estrecha interacción con la adrenalina y la dopamina que cumplen un papel tranquilizante o producen lo contrario. Ciertas hormonas tienen un rol destacado en todo lo relativo a la ansiedad como el cortisol y la ACTH (Bustamante, 2013, pp.6-9).

Entre los factores biológicos, los elementos genéticos se han encontrado muy ligados a los trastornos de ansiedad, con mayor tendencia el trastorno obsesivo compulsivo, el de pánico y la fobia social. Como ocurre con la mayor parte de los trastornos psicológicos, no se ha encontrado un gen único que genera la ansiedad, sino un conjunto de pequeños aportes de varios genes compuestos por varias áreas de los cromosomas que convierten en vulnerables a padecer de ansiedad a las personas (French, 2013, s.p.). La ansiedad también se relaciona con circuitos cerebrales como los sistemas Gaba, neurotransmisor principal en el sistema nervioso central que reduce la excitabilidad neuronal a lo largo del sistema nervioso y regula el tono muscular. El sistema CRF activa el eje hipotalámico, hipofisario y adrenal, y tiene grandes efectos sobre las áreas del cerebro relacionadas con la ansiedad, tomando en cuenta el sistema límbico que incluye el hipocampo, la amígdala y la corteza prefrontal, y los sistemas noradrenérgico y sertoninérgico que actúan de forma bilateral para controlar el sueño y el insomnio. Los factores biológicos pueden alterar el comportamiento de los individuos e inducir a problemas en las relaciones sociales, laborales y personales, para lo cual es importante buscar ayuda profesional para un tratamiento 
adecuado (Galletero, Guimón, Echeburúa, Yllá y González, s.f. pp.1-9).

Los estresores psicosociales como problemas relacionados con el ambiente social, con la enseñanza, así como los problemas laborales, económicos, de acceso a servicios médicos y relacionados con la interacción con el sistema legal, son factores detonantes de los trastornos psicosociales.

Los factores traumáticos como enfrentar acontecimientos importantes como accidentes, desastres naturales, asaltos, violaciones, torturas y secuestros suelen provocar serios daños biológicos cerebrales que se asocian a síntomas graves de ansiedad.

Los factores cognitivos y conductuales están compuestos por una serie de rasgos ligados al aspecto social. Las personas se muestran incómodas por causa de los pensamientos estresantes, los cuales pueden generar aceleración fisiológica -que responde al aumento de la presión sanguínea y de la frecuencia cardíaca o taquicardia-, secreción de cortisona, un estado de alerta, un flujo de pensamientos y reacciones más rápido; asimismo, altera su actuación y la percepción que tienen las demás personas sobre estos individuos. Estos acontecimientos producen que estas personas experimenten altos niveles de ansiedad y bajos niveles de atención hacia los demás, lo que trasciende negativamente en su participación social.

Otros elementos psicológicos que se consideran importantes son los factores de aprendizaje, como déficit atencional o la ansiedad condicionada que es la reacción ante una acción o estímulo. Los pacientes con fobia social pueden presentar ausencia de la amplia gama de algunas conductas o expresiones comportamentales, sean estas sexuales o no, que se consideran necesarias para el desarrollo de las relaciones interpersonales. Algunos esquemas de incorporación y proceso de la información llegan a tener un papel principal en la dirección que puede tomar la conducta de una persona. Ciertos procesos cognitivos, sean conscientes o inconscientes, como la memoria, la atención, el lenguaje, actuarían como causas cercanas a padecer niveles de ansiedad (Fernández, Sierra y Zubeidat, 2006, s.p.).

El sistema atencional es el encargado de detectar y controlar, mediante los mecanismos necesarios, los estímulos circunstanciales y sensoriales internos significativos para el estado motivacional del organismo. Aquí está muy ligada la ansiedad social considerada como una emoción adaptativa para la supervivencia del individuo, la cual prepara el organismo ante cualquier amenaza o riesgo originario del ambiente y le sirve al sujeto para actuar de un modo rápido y eficaz ante estos. Sin embargo, la detección de amenazas y peligros del ambiente por parte de los individuos a menudo es exagerada, ya que se identifica o estima una variedad de estímulos apenas amenazantes como muy peligrosos. Se considera que los modelos cognitivo-comportamentales existentes, el hipotético deductivo y el observacionalexperimental, conceden un rol importante en el apartado atencional (Fernández, Sierra y Zubeidat, 2006, s.p.).

Asimismo, las investigaciones se han fascinado por la posibilidad de relacionar la memoria en individuos con trastornos de ansiedad, de manera que estos retendrían la información amenazante relativa a sus miedos y temores. Hay dos teorías que sustentan esta idea: la teoría de la red del estado del ánimo y la memoria de Bower y la teoría de 
los esquemas de Beck. De acuerdo con estas hipótesis, los sujetos ansiosos suelen presentar una mejor memoria para la información asociada a la ansiedad (Cano-Vindel, 1999, pp.6-7). Los individuos con tendencia a desarrollar trastornos depresivos poseerían una debilidad cognitiva en forma de esquemas cognitivos negativos acerca de ellos o ellas mismas, el mundo y el futuro, obtenidos a partir de experiencias pasadas negativas y traumáticas, que pueden continuar disfrazadas hasta que ciertos hechos estresantes, similares a los implicados en la formación del esquema, se hicieran responsables de su aceleración. Los sujetos con vulnerabilidad a desarrollar trastornos de ansiedad dispondrían de una serie de esquemas referentes a la amenaza, sobre todo en torno a tres temas: aceptación, competencia y control.

Dentro de los factores familiares que inciden en la ansiedad, se mencionan ciertos estilos de crianza, por ejemplo, el control, la sobreprotección excesiva, la intrusión, el abandono, la indiferencia y el escaso contacto con experiencias sociales. Los mensajes comportamentales pueden acarrear sentimientos de disgusto en las situaciones sociales y la manifestación de muestras de ansiedad por parte de algunos progenitores que, como modelos, incitan a que los hijos e hijas adquieran estos comportamientos mediante la observación. Otro factor importante dentro de los estilos de crianza inadecuados es la aprobación de pocas oportunidades de exposición en encuentros sociales, lo cual genera sentimientos negativos para poder actuar en dichas situaciones, valoradas como difíciles y complicadas. En estos casos, algunos padres y madres estarían permanentemente preocupados e influidos por la opinión de los demás, obstaculizando la sociabilidad, empleando a menudo la vergüenza como un procedimiento de control y disciplina, y evitando de esta manera con frecuencia las situaciones sociales. Otros acontecimientos como ser rechazado, ser hijo o hija únicos, aparición de padecimientos comunes como el acné, ser rechazados por el nacimiento de otros hermanos o hermanas que tengan lugar en el transcurso de la primera infancia y la adolescencia, pueden tener una participación determinante en el surgimiento de un trastorno de ansiedad.

El médico psiquiatra Juan Bonilla Torres, especialista del Hospital General de la Zona N. $^{\circ} 21$ del Instituto Mexicano del Seguro Social (IMSS), de la ciudad de León, menciona en el periódico Informador. $M x$, de Jalisco, México (10 de julio de 2014), que "los adultos jóvenes son el sector que está más expuesto a sufrir ataques de ansiedad, debido a que son inquietos, perfeccionistas, apresurados". Agrega que la mayoría de los pacientes que llega al servicio de emergencias son adultos jóvenes de sexo masculino y presentan síntomas como taquicardia, sudoración, náuseas, sensación de ahogo, hormigueo de manos y piernas, entre otros síntomas; en algunos casos, estos pacientes no saben decir lo que les está ocurriendo.

\section{La ansiedad y la adquisición de una segunda lengua}

En el momento en que algunos de los adultos jóvenes decidan o tengan que adquirir una segunda lengua, surge la incógnita si para algunos o algunas este proceso se pueda dar sin mayores problemas; sin embargo, otros 
individuos no obtendrán un buen desempeño y su aprendizaje se tropezará con un complejo de dificultades. Diversos investigadores del área de la enseñanza de las lenguas extranjeras han señalado que factores como las experiencias previas en el aprendizaje, la motivación, la ansiedad, los estilos de aprendizaje y las estrategias de estudio son clave en el desarrollo de la adquisición de una segunda lengua (Galindo, 2010, p.41).

Para aprender debe haber un mínimo de estrés. La tensión se evita cuando el sujeto se siente motivado para aprender y cuando una urgencia de comunicación está complacida al poder expresar sus ideas mediante lo aprendido. Poder comunicarse de forma eficiente y obtener los resultados deseados hace que el individuo esté en un estado de calma y ecuanimidad, lo que refuerza la confianza en sí mismo y mantiene la motivación durante el proceso de aprendizaje.

Se ha venido observando a través del tiempo que la ansiedad se presenta en la clase de lengua extranjera en individuos con las habilidades necesarias para su adquisición, pero quienes, a pesar de esto, se notan incómodos o molestos, lo que les dificulta el uso de este segundo idioma. Esta dificultad puede ser más evidente cuando se requiere destacar la destreza oral. Los psicólogos MacIntyre y Gardner, citados por García Galindo (2010), han definido el mecanismo por el que puede surgir la ansiedad; en consecuencia, un estudiante en sus primeras etapas de aprendizaje podría encontrarse con muchas dificultades que comenzarían por la imposibilidad para reproducir los sonidos de la lengua meta hasta los problemas propios de la adquisición de una nueva gramática.
Si estas primeras experiencias ponen irritable o nervioso al sujeto y, además, se siente incómodo cuando se equivoca, se desarrolla la denominada "ansiedad de situación" (García Galindo, 2010, p.47). Si sucede repetidamente, el individuo comienza a asociar la activación de ansiedad ("anxiety arousal" en inglés) con la lengua extranjera y con su aprendizaje. Este mecanismo se va retroalimentando, pues la persona comienza a creer que va a sentirse en el mismo estado de ansiedad en experiencias de aprendizaje de una segunda lengua. La ansiedad entonces se difunde a partir de experiencias negativas y repetitivas ante el aprendizaje de la lengua extranjera.

\section{La afectividad, la ansiedad y el aprendizaje de una lengua extranjera}

Para efectos de este estudio se considera como afectividad la "capacidad de reacción de un sujeto ante los estímulos que provienen del medio externo o interno, cuyas principales manifestaciones son los sentimientos y las emociones" (WordReference.com). La afectividad está determinada por la facultad de experimentar interiormente las realidades exteriores y transformar en vivencia interna algún contenido de juicio.

La noción de afectividad incluye todos los elementos de los sentimientos, la emoción, las vivencias y los estados de ánimo que condicionan la conducta del ser humano y que median en el aprendizaje de los idiomas pues están presentes en la realidad del aula (Arnold, 2005). Entender la labor que ejerce el componente afectivo en el proceso de enseñanza de un individuo es preocuparse por la forma de solucionar 
problemas originados por las emociones negativas y, por tanto, crear reacciones positivas y facilitadoras que proporcionarán una luz a los y las docentes para comprender mejor la forma de aprendizaje de sus estudiantes y aplicar los métodos más eficaces (Arnold, 2005).

Los estudiantes comienzan a ser percibidos como seres extraordinarios, porque llevan consigo una variedad de elementos internos, intereses y habilidades. Además, guardan experiencias personales que se sumarán a sus estilos de aprendizaje cuando surgen diferentes modelos de enseñanza como el constructivismo, el holístico y los distintos enfoques como el humanístico y las metodologías como el interaccionismo social, en donde se da importancia al entorno familiar y cultural y al contexto en la adquisición del lenguaje en edad temprana.

Cuando los estudiantes descubren que los mecanismos cotidianos de defensa ante un peligro no funcionan, se producen consecuencias negativas para el aprendizaje de una lengua extranjera. Cuando se percibe que lo rutinario requiere de un esfuerzo laborioso, esto provocará en el individuo estrés, perturbación e incluso miedo. Estos sentimientos no son ideales para el aprendizaje en general y menos aún para la adquisición de una lengua extranjera que, en muchos casos, no viene a representar relevancia para el individuo pues carece de función social o institucional.

En el área del aprendizaje, la ansiedad debe entenderse, por una parte, como un estado, una emoción que es normalmente evidente en eventos precisos; por otra parte, la ansiedad como rasgo está almacenada oculta o disfrazada en el individuo (Moreno, 2004). Tomemos como ejemplo situaciones evaluadoras; entonces, la ansiedad tendrá dos componentes: uno emocional que se representa por medio de las reacciones fisiológicas, como la sudoración, la aceleración de los latidos y los movimientos incontrolables de pies, manos u otras partes del cuerpo. El otro componente es el cognitivo concerniente a las preocupaciones sobre el éxito y la forma de actuar, siendo este último el que más directamente interfiere en el aprendizaje del alumno.

Así también, los individuos con una escasa preparación para enfrentar evaluaciones, especialmente presentaciones orales, son más susceptibles a experimentar gran ansiedad debido a un aumento de inseguridad la cual ya muchas veces está implicada. Aquellos que constan de una mejor organización para encarar situaciones orales en la lengua extranjera serán más confiados y, por ende, no experimentarán tanta ansiedad.

Existen tres niveles por los cuales la ansiedad obstruye el aprendizaje. Según Tobías (1994), en el primero, la ansiedad impide el procesamiento previo y eficaz de los nuevos conocimientos que se están adquiriendo; entonces, el individuo presenta dificultades para entender y organizar. En el segundo, la ansiedad obstruye con el procesamiento; es decir, cuando se solicita al estudiante aplicar la nueva información en situaciones específicas, es incapaz de recordar lo que ha aprendido, o bien, utiliza de forma ineficaz las destrezas para resolver dificultades. El tercer nivel, se refiere a la producción, la cual se puede disipar antes de que el estudiante la aprenda; habrá casos de alumnos que serán capaces de demostrar su conocimiento en el momento de aprenderlo pero tendrán dificultades para expresarlo en actividades posteriores. 


\section{La ansiedad en los estudiantes de un idioma extranjero}

Cada competencia comunicativa del estudiante depende de su actitud personal hacia el nuevo idioma. No obstante, existen también algunos factores que interfieren en este proceso de aprendizaje, tales como las diferencias personales, el entorno en el que estudian, los factores afectivos y los conocimientos previos que cada uno de ellos trae a la clase. A raíz de estas ideas, en las últimas décadas, diversos investigadores, como Brown (1980), Krashen (1981) y Scovel (1978), entre otros, han estudiado los factores afectivos implicados en el proceso de enseñanza de un idioma extranjero; se menciona la ansiedad como uno de los factores causantes de fallas en el aprendizaje de la lengua como: producción de errores gramaticales y de pronunciación de los estudiantes, y la dificultad para entender el vocabulario que el profesor les está enseñando.

Del mismo modo, Aida (1994), Horwitz, Horwitz y Cope (1986), MacIntyre y Gardner (1989) y Phillip (1992), entre otros, explican en sus estudios de qué manera la ansiedad afecta el desempeño de los estudiantes en la clase de lengua y cómo la comprensión de sus causas ayuda a reducir y mejorar el rendimiento de los aprendices.

\section{La Escala de Ansiedad de medición en un Aula de Lengua Extranjera (FLCAS)}

Horwitz, Horwitz y Cope (1986) diseñaron una escala especial para identificar la ansiedad en el aprendizaje de una lengua extranjera. En este instrumento, se enfoca la ansiedad como la combinación de la intranquilidad a la hora de comunicarse y el temor acerca de la evaluación negativa por parte de los compañeros. Para la creación de esta herramienta estos autores consideraron los informes presentados por algunos estudiantes, experiencias clínicas psicológicas e instrumentos creados anteriormente para medir la ansiedad. Es una escala Likert formada por 33 piezas, que se evalúa a partir de 1 (Totalmente en desacuerdo, TD) a 5 (Muy de acuerdo, SA).

No obstante, antes de usar la herramienta para medir el nivel de ansiedad en los estudiantes de inglés para otras carreras en la UNA, fue necesario modificarla de manera que se incluyeron solo 13 preguntas, pero con las mismas unidades de medida: Muy de acuerdo, De acuerdo, Ni de acuerdo ni en desacuerdo, En desacuerdo y Muy en desacuerdo. Un cambio que se realizó debido a que la original exigía a los estudiantes más tiempo para responder.

\section{Las estrategias para reducir la ansiedad}

Según Rubio (2004, p.159), si un profesor necesita reducir la ansiedad de los alumnos durante las clases orales, debe eliminar la causa cuando sea posible o ayudar a los estudiantes para que la enfrenten. Una acción que puede realizar es mejorar su actitud hacia lo que enseña y crear un ambiente de aula positivo con el uso de técnicas en los niveles fisiológico y psicológico que inspiren sensaciones de relajación y tranquilidad; además, puede modificar su diálogo interno o aquellas emociones que puedan afectar la disposición que tengan hacia la tarea y sus resultados hacia el aprendizaje.

Una tarea que puede realizar exitosamente si trata al estudiante con respeto, le ayuda a invalidar los sentimientos de 
inseguridad y crea un ambiente de cooperación y no de competición, entre otros. Así mismo, si no lo presiona a hablar antes de que sienta que es capaz de hacerlo, e implementa una práctica de corrección de errores que no sea amenazante durante las lecciones. De esta forma, ayuda a que exista en los alumnos un mínimo nivel de estrés cuando tienen que usar el inglés para comunicarse de forma oral con sus compañeros de clase o con la profesora, se sientan motivados para aprender y satisfechos al poder expresar sus ideas en esta lengua extranjera.

\section{Metodología}

Esta investigación se ocupa de la ansiedad como un factor emocional que afecta la participación de los estudiantes durante las clases orales de inglés como lengua extranjera. Tiene como participantes a 29 alumnos de 2 grupos de Inglés I para otras carreras en la UNA; se seleccionaron tres técnicas de recolección de datos. En primer lugar, la escala de ansiedad FLCS (Lengua Extranjera Aula Escala de Ansiedad) de Howitz, Horwitz y Cope (1986); esta herramienta sirvió de diagnóstico, ya que se utilizó para conocer el nivel de ansiedad que estaban experimentando los estudiantes durante las clases orales de inglés, por medio de las respuestas dadas a trece preguntas. En segundo lugar, una herramienta de observación que fue completada por las dos profesoras encargadas de impartir esos cursos de lengua y, finalmente, se aplicó a los estudiantes un cuestionario que incluyó doce preguntas que tenían como objetivo conocer los puntos de vista de cada uno de ellos sobre la efectividad de las estrategias implementadas durante cuatro meses continuos y que tenían la idea de reducir el nivel de ansiedad que habían demostrado cuando se aplicó el instrumento de diagnóstico.

Es una investigación de tipo cualitativo y con un diseño cuasi-experimental. Una investigación cualitativa que, según Khan (2006), “describe los acontecimientos y personas científicamente sin el uso de los números numéricos" (p.79) y con un diseño cuasiexperimental, ya que tiene el propósito de determinar si el conjunto de estrategias de enseñanza recomendadas fue eficaz para reducir el nivel de ansiedad en estos 29 estudiantes cuyas edades se detallan seguidamente en la Tabla 1:

Tabla 1. Edades de los estudiantes

\begin{tabular}{cc}
\hline Ages & N. $^{\circ}$ of Students \\
\hline $17-19$ & 24 \\
$20-22$ & 4 \\
$23-25$ & 0 \\
$26-27$ & 1 \\
\hline
\end{tabular}

Como se ilustra en esta Tabla 1, la mayoría de los estudiantes tienen entre 17 y 19 años de edad, y dentro de este rango de edad en Costa Rica muchos de ellos acaban de terminar la etapa secundaria. Así mismo, la mayoría de los estudiantes ha estudiado inglés (en la escuela primaria y en la secundaria) por cerca de 11 años en las instituciones públicas, y el mismo número ha tenido buenas experiencias durante el aprendizaje de esta lengua extranjera.

\section{Análisis y resultados}

Una vez recopilada y analizada la información, los resultados que se obtuvieron son los siguientes: 


\section{La escala FLCA como instrumento de diagnóstico}

En los cursos de Inglés para otras Carreras en la Universidad Nacional los profesores a cargo acostumbran diseñar actividades de clase que tienen como objetivo principal hacer que los estudiantes no solo lean y escriban en este idioma extranjero, sino que escuchen y hablen también. No obstante, y en el caso de este grupo específico, cuando era necesario hacerlos interactuar en inglés en las diferentes actividades orales planeadas para cada clase, en su mayoría se conducían de forma ansiosa y nerviosa y no querían hablar.

Por esta razón y después de varios meses de percibir estas reacciones en ellos, las profesoras se reunieron y decidieron aplicar la escala FLCAS para diagnosticar el nivel de ansiedad que podrían estar experimentando; los resultados más importantes se describen a continuación:

Gráfico 1. Respuestas dadas por los estudiantes a la pregunta $\mathrm{N} .^{\circ} 1$ en la escala FLCA usada como diagnóstico

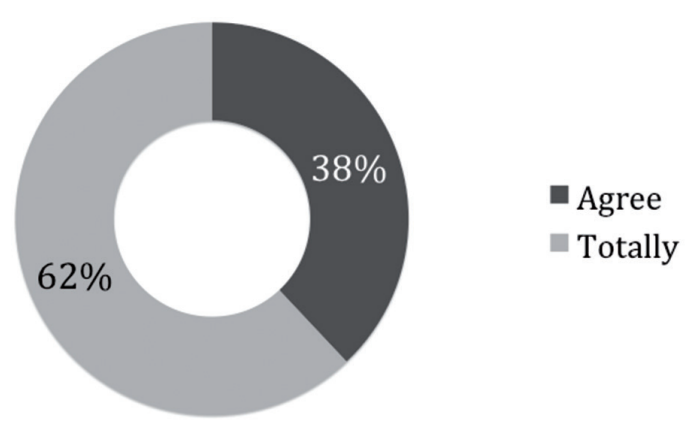

\section{En clases me siento tímido(a) y avergonzado(a) cuando hablo en inglés}

Como se muestra en este gráfico, la mayoría de los estudiantes se sienten tímidos y avergonzados (62 \%) al hablar en inglés durante las clases de este idioma, lo que demuestra que efectivamente sufren de ansiedad cuando tienen que realizar actividades orales en esta segunda lengua.

La pregunta $\mathrm{N}^{\circ} 2$ de esta misma herramienta sobre si se sienten temerosos cuando no entienden lo que la profesora dice en inglés durante las lecciones dio un resultado de un $62 \%$ que indica que está de acuerdo con esta afirmación y solo un 38\% está en desacuerdo. (Ver Gráfico 2).

Gráfico 2. Respuestas emitidas en la pregunta $\mathrm{N}^{\circ} 2$ de la escala FLCA usada como diagnóstico

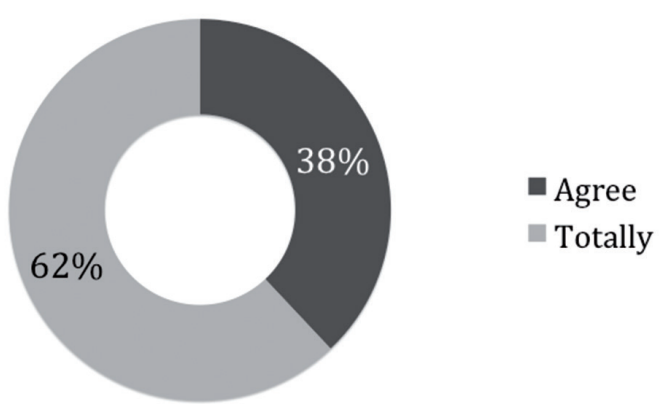

\section{Me siento temeroso(a) cuando no entiendo lo que la profesora trata de decir en inglés}

Las respuestas muestran un comportamiento que podría estar relacionado con la falta de confianza que los estudiantes tienen sobre sus habilidades en inglés y que afecta incluso su comprensión de lo que dice el profesor. 
Otro de los ejemplos que se seleccionaron como muestra de las respuestas dadas por los estudiantes en la escala FLCA usada como diagnóstico es la pregunta N. ${ }^{\circ} 6$ sobre si sienten pánico cuando tienen que hablar espontáneamente en inglés, a lo que los estudiantes respondieron de la siguiente manera:

Gráfico 3. Respuestas emitidas en la pregunta $\mathrm{N} .{ }^{\circ} 6$ de la escala FLCA usada como diagnóstico

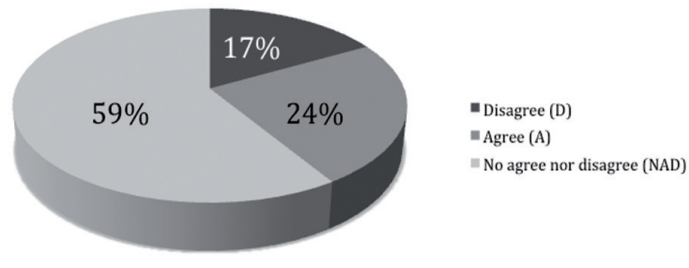

\section{Siento pánico cuando tengo que} hablar espontáneamente en inglés

Cuando los estudiantes tienen que hablar de forma espontánea, la mayoría de ellos no está de acuerdo con esta situación, porque a veces prefieren tener algo de tiempo para prepararse antes de participar en cualquiera de las actividades orales.

Gráfico 4. Respuestas emitidas en la pregunta $\mathrm{N}^{\circ} 9$ de la escala FLCA usada como diagnóstico

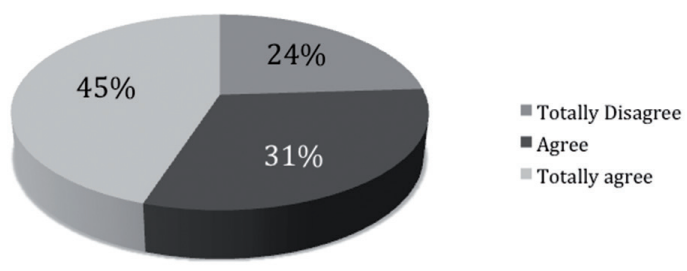

\section{Tengo miedo de que los otros estudiantes se rían de mí cuando hablo en el idioma extranjero}

En las respuestas a la pregunta N. ${ }^{\circ}$ 9 de la escala FLCA (Gráfico 4), el 45\% de los estudiantes confirmó sentir miedo de hablar en inglés, ya que piensan que sus compañeros de clase se reirían de ellos. Un factor emocional que necesita ser reducido en la clase si queremos hacer que interactúen con sus compañeros con más confianza.

\section{La herramienta de observación de las profesoras}

La segunda herramienta que se utilizó para recopilar los datos de este trabajo es una lista de cotejo que incluía 9 preguntas sobre la efectividad en el uso de las estrategias para reducción de la ansiedad en los estudiantes durante las clases orales de inglés, recomendadas por Galindo (2010). Las dos profesoras a cargo de los grupos seleccionaron estudiantes al azar y, según lo que observaban, determinaban si la aplicación de estas técnicas era efectiva, no efectiva o si era necesario reforzar cada día de estos cuatro meses en que los estudiantes estuvieran inmersos en las actividades orales durante las lecciones de inglés, cuyos resultados de observación se detallan a continuación:

La estrategia relativa a detener sus sentimientos negativos (No puedo hacerlo, Esto es muy difícil de hacer, No puedo aprender, etc.) fue efectiva solamente al final de los cuatro meses. Se considera necesario reforzar su uso en futuras clases orales de inglés con estos alumnos. 
Sin embargo, las estrategias para reducir la ansiedad en otro idioma como: tratarlos con respeto, dando posibilidades de expresar sus preocupaciones, no empujar a hablar antes de que se sientan capaces de hacerlo, la creación de un marco de cooperación y no la competencia, las prácticas indirectas de corrección de errores, y la creación de un ambiente de relajación y aprendizaje de confianza, se consideran eficaces para reducir la ansiedad en este grupo de estudiantes. Al final del semestre, fueron más entusiastas a participar e interactuaron con sus compañeros de clase; además, se trabajó de manera positiva en las actividades

\section{La Escala de Likert para medir la efectividad de las estrategias para la reducción de la ansiedad recomendadas por Rubio (2004)}

Una vez concluido el periodo de cuatro meses que se planeó para la aplicación de las Estrategias de Reducción de la Ansiedad en las clases orales de inglés con la población seleccionada, se distribuyó entre los estudiantes una Escala de Likert que incluía preguntas que pretendían conocer sobre su estado de ánimo una vez que se implementó el uso de estas estrategias durante las lecciones.

En este instrumento, el 100\% de los estudiantes (29 en total) contestó que está totalmente de acuerdo en cuanto a una mejora en su nivel de ansiedad al utilizar estrategias como tratarlos con respeto, darles posibilidades de expresar sus preocupaciones, no obligarlos a hablar hasta que no se sintieran seguros, la creación de un ambiente de cooperación y no de competencia, y el uso de prácticas indirectas de corrección de errores entre otros. Además, es fácil percibir una mejora en su actitud hacia el idioma, ya que 12 de ellos (41.3\%) dicen sentirse más positivos y con un sentimiento de seguridad en sí mismos cuando hablan inglés. En cuanto a las actividades orales que se planean para las lecciones, solo 6 de ellos (20\%) siguen sintiendo timidez y un poco de vergüenza al hablar en inglés. Estos resultados demuestran la eficacia de las estrategias aplicadas durante las lecciones y coinciden con los resultados y las observaciones hechas por las docentes.

Por otro lado, en cuanto a la estrategia relativa a detener sus sentimientos negativos (No puedo hacerlo, Esto es muy difícil de hacer, No puedo aprender, etc.), un $75 \%$ de ellos concuerda en la necesidad de exponerlos por más tiempo a este tipo de técnica que les ayudaría a mejorar estos sentimientos y sentir más seguridad a la hora de hablar.

\section{Conclusiones y recomendaciones}

A continuación, se presenta una lista de conclusiones y recomendaciones que se obtienen de los resultados finales de esta investigación y que están relacionadas con el objetivo de encontrar posibles técnicas o estrategias para la reducción de la ansiedad para ayudar a los estudiantes a sentirse más seguros al utilizar el idioma inglés para comunicarse con sus compañeros de clase o con sus profesores.

1. Conocer los factores emocionales que podrían afectar la participación de los estudiantes en las clases orales ayudará a los maestros a conocer las limitaciones de aprendizaje y a 
utilizar actividades que sean apropiadas según el nivel de lengua de sus alumnos.

2. Es necesario que los docentes tengan en cuenta las razones por las que los estudiantes se sienten ansiosos en las clases orales de inglés si quieren mejorar su participación en las diferentes actividades que se han programado para el curso. Las estrategias de enseñanza que se utilicen durante las lecciones deben ser diseñadas según las necesidades emocionales de cada uno de los estudiantes.

3. La aplicación de estrategias para la reducción de la ansiedad en las clases orales de inglés son de gran impacto para mejorar las habilidades orales de los estudiantes.

4. Las estrategias que tienen relación con los sentimientos negativos en los estudiantes sobre sus capacidades de hacer las cosas en inglés requieren de un mayor esfuerzo y más tiempo de aplicación.

En consecuencia, las estrategias para reducción de la ansiedad recomendadas por Rubio (2004) resultaron eficaces para que los estudiantes durante las clases orales de inglés se sientan más seguros al utilizar este idioma extranjero al interactuar con sus compañeros de clase y con sus profesoras, por lo que se recomienda su uso por profesores que tengan situaciones similares en sus salones de clase.

\section{Bibliografía}

Aida, Y. (1994). Examination of Horwitz, Horwitz, and Cope's construct of foreign language anxiety:
The case of students of Japanese. The Modern Language Journal, 78, 155-168.

Alrabai, F. (2014). The influence of teacher's anxiety-reducing strategies on learners' foreign language anxiety. Retrieved: DOI: 10.1080/17501229.2014.890203 American Psychological Association. (2015). Anxiety. Retrieved: http://www.apa.org/topics/anxiety/

Arnold, J. (2005). El aula de ELE: un espacio afectivo y efectivo. Recuperado de: http://cvc.cervantes.es/ensenanza/biblioteca_ele/ publicaciones_centros/PDF/munich_2005-2006/03_arnold.pdf

Anxiety Disorders Association of America (2002). About ADAA: Goals and objectives. Retrieved September 10, 2002 Recuperado de: http://wwwadaaorg/aboutadaa/ goalsobjectives.

Beltrán, J., Bueno, J.A. (1995). Psicología de la Educación, p.239. Recuperado de: https://books.google.es

Best, J. and Kahn, J. (2006). Research in Education. 10 edition Pearson Education Inc. USA. Recuperado de: http:// ebook.stepor.com/book/research-ineducation-10th-edition-66484-pdf. html

Brown, D. (1980). Principles of language learning and teaching. Prentice Hall Regents.

Bustamante, Ricardo. (2013). X Congreso Internacional de Salud y Desarrollo de Adolescentes y Jóvenes "Comprometidos siempre". Ansiedad en adolescentes y jóvenes. Aspectos Genéticos y Epigenéticos. Recuperado de: http://www.codajic. org/sites/www.codajic.org/files/Ansiedad\%20-Bustamante.pdf

Cano-Vindel, A. (1999) Procesos cognitivos en el trastorno de ansiedad gene- 
ralizada, según el paradigma del procesamiento de la información. Revista electrónica de psicología, vol. 3, n. ${ }^{\circ}$, enero 1999, issn 1137-8492 Recuperado de: http://www.researchgate.net/ profile/Antonio_Cano-Vindel/publication/230577035_Procesos_cognitivos_ en_el_trastorno_de_ansiedad_generalizada_segn_el_paradigma_del_ procesamiento_de_la_informacin./ links/09e4150196059c1212000000. pdf

Chomsky, N. (1965). Aspects of the Theory of Syntax. Cambridge, Mass. MIT Press.

Fernández, A., Sierra, J., y Zubeidat, I. (2006). Factores asociados a la ansiedad y fobia social. Revista Mal Estar e Subjetividade. Recuperado de: http://pepsic.bvsalud.org/ scielo.php?script=sci_arttext\&pid $=$ S1518-61482006000200010

French, M. (2013). Ansiedad: factores biológicos y tratamientos. Recuperado de: http://suite101.net/article/ ansiedad-factores-biologicos-y-tratamientos-a72422\#.VaAs0xt_Oko

Galletero, J., Guimón, J., Echeburúa, E., Yllá, L. y González J. L. (s.f.). Monografias de Psiquiatría. Vol. 1, No. 4. Etiología de la Ansiedad. Recuperado de: http://www.psicoter.es/pdf/etiolog_ansiedad.pdf

García, G. (2010). La Ansiedad ante el Aprendizaje de una Lengua Extranjera. Revista de la Educación en Extremadura. D.L. BA 000098. 2010. Recuperado de: http://www. anpebadajoz.es/autodidacta/autodidacta_archivos/numero_5_ archivos/4_g_g_galindo.pdf

Gardner, R. C. y MacIntyre, P. D. (1993). A student's contribution to second language learning. Part II: Affective variables. Language
Teaching, 26, 1-11. Recuperado de: http://faculty.cbu.ca/pmacintyre/ research_pages/journals/affect_ 12learn1993.pdf

Horwitz, E., Horwitz, M. y Cope, J. (1986). Foreign language classroom anxiety. The Modern Language Journal, 70, 125-132

Krashen, S. (1981). Second Language Acquisition and Second Language Learning. California: University of Southern California, Pergamon Press Inc.

MacIntyre, P. y Gardner, R. (1989). Anxiety and second language learning: Toward a theoretical clarification. Language Learning, 39, 251-275.

MacIntyre, P., y Gardner, R. (1991a). Language anxiety: Its relationship to other anxietie and to processing in native and second languages. Language Learning, 41, 513-534.

Mena, T. (2013). Factores afectivos que inciden en el aprendizaje de una lengua extranjera. Universidad de Oviedo. Recuperado de: http:// digibuo.uniovi.es/dspace/bits tream/10651/18314/6/TFM_\%20 MenaBenet.pdf

Moreno-García, C. (2004). La enseñanza de español como lengua extranjera. Dirección URL: https://books. google.es

Oxford, R.L. (1990). Language Learning Strategies: What Every Teacher Should Know. Boston, MA: Heinle \& Heinle.

Pearson Longman. (2013). Teacher`s Guide to the Common European Framework. Recuperado de: http:// lcci.hu/hun/wp-content/uploads/cefguide.pdf

Periódico El Informador (10 de julio, 2014), Guadalajara, Jalisco, México. Recuperado de: http://www. 
informador.com.mx/suplementos/2014/541350/6/adultos-joveneslos-mas-propensos-a-ataques-deansiedad.htm

Phillips, E. (1992). The Effects of language anxiety on students' oral test performance and attitudes. The Modern Language Journal, 76, 14-26.

Poveda J. (2005). Afectividad. Arvo. net Recuperado de: http://arvo.net/ conceptos-frecuentes-en-filoso/afectividad/gmx-niv590-con12284.htm

Rubio, F. (2004). La ansiedad en el aprendizaje de idiomas. Huelva: Universidad de Huelva. Recuperado de:http://www.uhu.es/ fernando_rubio/universidad/INVESTIGACION/my_publications_ to_download_archivos/2004\%20 univ\%20huelva $\% 20$ ansiedad $\% 20$ aprend\%20idioma.pdf

Saito, Y., Horwitz, E. y Garza, T. (1999). Foreign language reading anxiety. The Modern Language Journal, 83, 202-218. Recuperado de: http://onlinelibrary.wiley.com/doi/10.1111/00267902.00016/abstract

Savignon, S. (1972). Communicative competence: an experiment in foreign language teaching. Philadelphia: Center for Curriculum Development. Recuperado de: http:// eric.ed.gov/?id=ED063835
Scovel, T. (1978). The effect of affect on foreign language learning: A review of the language anxiety literature. Language Learning, 28, 129-142.

Spielberger, C. (1983). Manualfor the state-trait anxiety inventory. Palo Alto, CA: Consulting Psychologists Press. Recuperado de: https://vanachinnappa.wordpress.com/2014/02/19/ anxiety-and-learning/

Tobias, S. (1994). Interest, Prior Knowledge, and Learning. Review of Educational Research, 45-46

Trujillo, M. (2010). Modelo cognitivoconductual. Recuperado de: http:// www.monografias.com/trabajos 82/ modelo-cognitivo-conductual/modelo-cognitivo-conductual.shtml

Valiente, C. (2011). Estudio neuropsicógico de funciones ejecutivas en religiosas meditadoras contemplativas. Madrid. Recuperado de: http:// eprints.ucm.es/13308/1/T33016.pdf

Virues, R. (2005). Estudio sobre ansiedad. Universidad Autónoma de Nuevo León. Monterrey, México. Recuperado de: http://www.psicologiacientifica.com/ansiedad-estudio

White, C. (2003). Language Learning in Distance Education. Cambridge: Cambridge University Press. 


\section{Anexos}

\section{Anexo 1}

\section{Universidad Nacional de Costa Rica (UNA) Inglés Integrado \\ I Students' Survey}

Dear student,

1. Personal information:

a) Age:

b) Gender: F ( ) M ( )

c) Years studying English:

d) Elementary or Higher Education Institution:

e) Personal experience in the Learning of English:

Very good: () Good:( ) Regular: () Bad: () Very bad: ()

Explain why you say so:

d) Major:

2. La escala FLCA como instrumento diagnóstico

\begin{tabular}{|c|c|c|c|c|}
\hline 1: (TD) & 2: (D) & 3: (NA/D) & 4: (A) & 5: (TA) \\
\hline Totally disagree & Disagree & Neither agree or disagree & Agree & Totally agree \\
\hline
\end{tabular}

Instructions: Please read each statement carefully and indicate how strongly you agree by checking in the corresponding number 


\begin{tabular}{|c|c|c|c|c|c|}
\hline & TD & $\mathbf{D}$ & N.A/D & $\mathbf{A}$ & TA \\
\hline & $\mathbf{1}$ & 2 & 3 & 4 & 5 \\
\hline $\begin{array}{l}\text { 1. It frightens me when I don't understand what the } \\
\text { teacher is saying in English. }\end{array}$ & & & & & \\
\hline $\begin{array}{l}\text { 2. I think the other students are better than me during } \\
\text { the English class. }\end{array}$ & & & & & \\
\hline 3. I often feel like not going to my language class. & & & & & \\
\hline $\begin{array}{l}\text { 4. I feel at ease when I need to communicate in English } \\
\text { with the Teacher or with native speakers. }\end{array}$ & & & & & \\
\hline $\begin{array}{l}\text { 5. I start to panic when I have to speak without prepara- } \\
\text { tion in the English class. }\end{array}$ & & & & & \\
\hline $\begin{array}{l}\text { 6. In class I get nervous and tend to forget topics, vo- } \\
\text { cabulary or Expressions that I know in English. }\end{array}$ & & & & & \\
\hline $\begin{array}{l}\text { 7. I look for opportunities outside the English class to } \\
\text { practice all the Contents that I learn during the English } \\
\text { lessons. }\end{array}$ & & & & & \\
\hline $\begin{array}{l}\text { 8. I feel overwhelmed when my English teacher corrects } \\
\text { me in front of my classmates. }\end{array}$ & & & & & \\
\hline $\begin{array}{l}\text { 9. I am afraid that the other students will laugh at me } \\
\text { when I speak the foreign language. }\end{array}$ & & & & & \\
\hline $\begin{array}{l}\text { 10. I enjoy very much the oral activities that the teacher } \\
\text { uses in the English class such as: role plays, pair work or } \\
\text { group conversations, Oral presentations, videos, among } \\
\text { others. }\end{array}$ & & & & & \\
\hline 11. I feel happy when I’m studying English. & & & & & \\
\hline $\begin{array}{l}\text { 12. The English classes that I have received before help } \\
\text { me to work well during the English classes. }\end{array}$ & & & & & \\
\hline
\end{tabular}

(Modified version from: The Foreign Language Classroom Anxiety Scale (FLCAS). (Horwitz et al., 1986) (The original includes 38 questions to select). A Likert scale is used. 


\section{Anexo 2}

\section{Universidad Nacional (UNA) Teachers Observation Checklist}

Effectiveness of the application of the Language anxiety-reducing strategies Name of the student:

1. Helping students to stop negative feelings that produce ansiety. (I can`t do it, this is very difficult to do, my classmates are going to laugh on me, I can`t learn it)

Effective

Non effective

To be reinforced

2. Reducing the anxiety in the students by treating him/her with respect and giving possibilities of expressing his/her worries.

Effective

Non effective

To be reinforced

3. Not pushing the student to talk before he/she feels able to do it

Effective

Non effective

To be reinforced

4. The creation of an environment of cooperation and not a competition

Effective

Non effective

To be reinforced

5. The indirect practice of error correction

Effective

Non effective

To be reinforced

6. A relax and trustful Learning environment

Effective

Non effective

To be reinforced

7. Communicative activities in pairs or small groups Effective Non effective

To be reinforced

8. Classroom activities that take into account the students emotional needs. Effective Non effective

To be reinforced

9. Classroom activities that take into account the students` learning styles Effective Non effective To be reinforced

10. The stimuli of the students'autonomy

Effective Non effective

To be reinforced

11. The impact of these Strategies to improve the learning process in the students

Effective

Non effective

To be reinforced 


\section{Anexo 3}

\section{Universidad Nacional de Costa Rica (UNA) Inglés Integrado I $2^{\text {nd }}$ Students`Survey}

Effectiveness in the use of the Language anxiety-reducing strategies in $\mathrm{My}$ English Classes

Dear student,

\begin{tabular}{|c|c|c|c|c|}
\hline 1: (TD) & $2:(D)$ & 3: (NA/D) & $4:(A)$ & 5: (TA) \\
\hline Totally disagree & Disagree & Neither agree or disagree & Agree & Totally agree \\
\hline
\end{tabular}

Instructions: Please read each statement carefully and indicate how strongly you agree by checking in the corresponding number

\begin{tabular}{|c|c|c|c|c|c|}
\hline & TD & D & NA/DD & $\mathbf{A}$ & TA \\
\hline & 1 & 2 & 3 & 4 & 5 \\
\hline $\begin{array}{l}\text { 1. After the teachers implemented some strategies during the oral } \\
\text { classes such as: making me feeling relax while listening, chang- } \\
\text { ing my inner dialogue, showing respect for each other, letting } \\
\text { me express my feelings, creating an environment in the class of } \\
\text { cooperation and not of competition, or correcting my mistakes } \\
\text { without making me feel threaten, it stills frightens me when I } \\
\text { don't understand what the teacher is saying in English. }\end{array}$ & & & & & \\
\hline $\begin{array}{l}\text { 2. After the teachers implemented some strategies during the oral } \\
\text { classes such as: making me feeling relax while listening, chang- } \\
\text { ing my inner dialogue, showing respect for each other, letting } \\
\text { me express my feelings, creating an environment in the class of } \\
\text { cooperation and not of competition, or correcting my mistakes } \\
\text { without making me feel threaten, I still thinking that the other } \\
\text { students are better than me during the English class. }\end{array}$ & & & & & \\
\hline $\begin{array}{l}\text { 3. After the teachers implemented some strategies during the oral } \\
\text { classes such as: making me feeling relax while listening, chang- } \\
\text { ing my inner dialogue, showing respect for each other, letting } \\
\text { me express my feelings, creating an environment in the class of } \\
\text { cooperation and not of competition, or correcting my mistakes } \\
\text { without making me feel threaten, I still feeling like not going to } \\
\text { my language class. }\end{array}$ & & & & & \\
\hline
\end{tabular}


4. After the teachers implemented some strategies during the oral classes such as: making me feeling relax while listening, changing my inner dialogue, showing respect for each other, letting me express my feelings, creating an environment in the class of cooperation and not of competition, or correcting my mistakes without making me feel threaten, I still feeling at ease when I need to communicate in English with the teacher or with native speakers.

5. After the teachers implemented some strategies during the oral classes such as: making me feeling relax while listening, changing my inner dialogue, showing respect for each other, letting me express my feelings, creating an environment in the class of cooperation and not of competition, or correcting my mistakes without making me feel threaten, I still feeling panic when I have to speak without preparation in the English class.

6. After the teachers implemented some strategies during the oral classes such as: making me feeling relax while listening, changing my inner dialogue, showing respect for each other, letting me express my feelings, creating an environment in the class of cooperation and not of competition, or correcting my mistakes without making me feel threaten, In class I still getting nervous and tend to forget topics, vocabulary or expressions that I know in English.

7. After the teachers implemented some strategies during the oral classes such as: making me feeling relax while listening, changing my inner dialogue, showing respect for each other, letting me express my feelings, creating an environment in the class of cooperation and not of competition, or correcting my mistakes without making me feel threaten, In class I still getting nervous and tend to forget topics, vocabulary or expressions that I know in English I feel more motivated to look for opportunities outside the English class to practice all the contents that I learn during the English lessons.

9. After the teachers implemented some strategies during the oral classes such as: making me feeling relax while listening, changing my inner dialogue, showing respect for each other, letting me express my feelings, creating an environment in the class of cooperation and not of competition, or correcting my mistakes without making me feel threaten, I still feeling afraid that the other students will laugh at me when I speak the foreign language. 
10. After the teachers implemented some strategies during the oral classes such as: making me feeling relax while listening, changing my inner dialogue, showing respect for each other, letting me express my feelings, creating an environment in the class of cooperation and not of competition, or correcting my mistakes without making me feel threaten, I still enjoying very much the oral activities that the teachers use in the English class such as: role plays, pair work or group conversations, oral presentations, videos, among others.

11. After the teachers implemented some strategies during the oral classes such as: making me feeling relax while listening, changing my inner dialogue, showing respect for each other, letting me express my feelings, creating an environment in the class of cooperation and not of competition, or correcting my mistakes without making me feel threaten, I still feeling happy when I`m studying English.

12. After the teachers implemented some strategies during the oral classes such as: making me feeling relax while listening, changing my inner dialogue, showing respect for each other, letting me express my feelings, creating an environment in the class of cooperation and not of competition, or correcting my mistakes without making me feel threaten, I keep thinking that the English classes that I have received before help me to work well during the English classes.

(Modified version from: The Foreign Language Classroom Anxiety Scale (FLCAS). (Horwitz et al., 1986) (The original includes 38 questions to select). A Likert scale is used. 